\title{
Smectite Crystallite Swelling Under High Pressure of Methane
}

Paweł P. Ziemiański ${ }^{1 *}$, Arkadiusz Derkowski ${ }^{1 *}$, Marek Szczerba ${ }^{1}$, Stephen Guggenheim ${ }^{2}$

${ }^{1}$ Institute of Geological Sciences, Polish Academy of Sciences, Research Centre in Krakow, Senacka St. No. 1, PL-31002 Krakow, Poland;

${ }^{2}$ Department of Earth and Environmental Sciences, University of Illinois at Chicago, Chicago, Illinois 60607, USA

\section{Grand Canonical Ensemble Monte Carlo simulations}

All simulations were performed using Towhee - Monte Carlo molecular simulation code ${ }^{1}$, using DX-1597-2-7 random numbers generator ${ }^{2}$. The clay model was represented by a rigid lattice, with a structure based on pyrophyllite ${ }^{3}$. The total charge of the montmorillonite layer was set to -0.58 per half unit cell to describe the Mtm sample ${ }^{4}$. The layer charge was introduced by substitution of $\mathrm{Al}^{3+}$ with $\mathrm{Mg}^{2+}$ in the dioctahedral sheet, maximizing the distance between $\mathrm{Mg}$ atoms ${ }^{5}$. Partial atomic charges and Lennard-Jones interatomic potential interactions of Mtm layers, interlayer cations, and $\mathrm{H}_{2} \mathrm{O}$ molecules, were described with ClayFF force field ${ }^{6}$. $\mathrm{CH}_{4}$ molecules and helium atoms were described with TraPPE-EH and TraPPE-UA force fields, respectively ${ }^{7}$. The standard Lorentz-Berthelot mixing rules were used to compute cross-interactions between atoms. Ewald summation was used for calculating the long-range electrostatic interactions contribution to potential energy. A cutoff of $11.0 \AA$ was used for electrostatic and Lennard-Jones interatomic interactions. Due to a large number of individual simulations, high complexity of model, and the long time to reach equilibration, no clay layer movement was allowed by fixing the atom positions in the 2:1 layer. The chemical potential of $\mathrm{CH}_{4}$ for each pressure step was calculated based on the $\mathrm{NpT}$ ensemble of $\mathrm{CH}_{4}$ gas in an empty box. The calculated values were: $-34.84,-29.17,-26.52$, $-25.31,-24.25,-23.55,-23.19$, and $-22.88 \mathrm{~kJ} / \mathrm{mol}$ for $1,10,30,50,80,110,130$, and $150 \mathrm{bar}$, respectively. 
Each production run was preceded by an equilibration run of at least $1.2 \times 10^{8} \mathrm{GCMC}$ steps in the

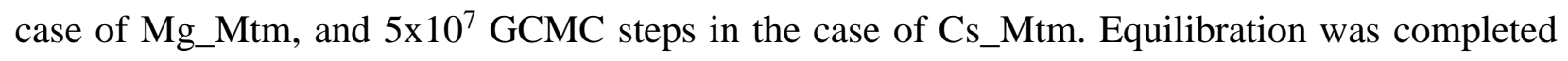
when the decreasing trend of the system potential energy reached a minimum plateau. The production run was set to $2 \times 10^{7}$ GCMC steps for both Cs_Mtm and Mg_Mtm models, divided into 20 segments. Averages were collected for the system potential energy, and the number of $\mathrm{CH}_{4}$ molecules in the simulation box. To calculate the available adsorption volume, helium-adsorption GCMC simulations were performed for the conditions of 1 bar of He pressure The average number of He atoms present in the interlayer was calculated from $2.5 \times 10^{7}$ GCMC-step simulations, and these simulations were preceded by $2.5 \times 10^{7}$ GCMC-step equilibration. 


\section{Calculations of disjoining pressure and free energy of swelling based on GCMC simulations}

The minimum of swelling free energy $(\Delta X)$ is related to the basal spacing of the simulated system remaining in thermodynamic equilibrium. $\Delta \mathrm{X}$ is calculated using eq. (1), which was derived for material with slit pores under constant temperature, a known chemical potential $(\mu)$ of the fluid, and normal-directed stress $^{8}$. This equation was applied successfully to describe $\mathrm{H}_{2} \mathrm{O}$-induced swelling in clay mineralsa ${ }^{9-11}$ :

$\Delta X=X\left(s_{z}\right)-X\left(s_{z}^{0}\right)=A \int_{S_{z}^{0}}^{s_{z}}\left[T_{Z Z}\left(s_{Z}^{\prime}\right)-T_{Z Z}^{\prime}\right] d s_{Z}^{\prime}$

where $\mathrm{X}\left(\mathrm{s}_{\mathrm{z}}\right)$ is free energy at $\mathrm{s}_{\mathrm{z}}$ basal spacing, $s_{z}^{0}$ is the reference basal spacing corresponding to maximally separated layers (15.0 $\AA$ in the present study), A is the basal surface area of the clay layer, $\mathrm{T}_{\mathrm{zz}}^{\prime}$ is constant external stress applied normal to the clay layer assumed to be equal only to bulk (isotropic) external pressure, with minus sign; $-\mathrm{P}_{\mathrm{b}}$, and $\mathrm{T}_{\mathrm{zz}}\left(\mathrm{s}_{\mathrm{z}}^{\prime}\right)$ is normal internal stress, defined as:

$T_{z Z}=-k T \frac{<N_{\text {int }}>}{V}+\frac{1}{A}\left\langle\left(\frac{\partial U}{\partial s_{z}}\right)_{\mu, T}\right\rangle$

where $\mathrm{k}$ is the Boltzmann constant, $\mathrm{T}$ is temperature, $\left\langle\mathrm{N}_{\text {int }}\right\rangle$ is an average number of all molecules $\left(\mathrm{CH}_{4}\right.$ and $\left.\mathrm{H}_{2} \mathrm{O}\right)$ and cations in the interlayer, $\mathrm{V}$ is volume of the interlayer [calculated as surface area (A) multiplied by $s_{z}$ reduced by the 2:1 layer thickness defined as a half of the average distance between centers of oxygen in tetrahedral sheet and closest atoms in the interlayer $(8.05 \AA)]$, $U$ is total potential energy of the system and $\mu$ is chemical potential. The partial derivative from eq. 2 was calculated by fitting a polynomial of $6^{\text {th }}$ order to the system potential energy (see Fig. S1). 
From mechanics, a system is in equilibrium with the environment when the disjoining pressure (П) acting on the internal surface of the layers has a negative slope and is equal to the external pressure applied to the clay layer ${ }^{9,10}$. П was calculated using eq. $3^{10}$ :

$\prod\left(s_{z}\right)=-T_{z Z}\left(s_{z}\right)-P_{b}$

where $\mathrm{P}_{\mathrm{b}}$ is a bulk fluid pressure (assumed in these calculations to be equal to $-\mathrm{T}_{z z}^{\prime}$ in eq. 1 , because no additional external stress pressure is applied normal to the clay layer).

Energy, entropy, and mechanical work contributions for the free energy of swelling were computed using eq. $4^{9-11}$, assuming zero shear forces:

$\Delta X=\left(\Delta E-E_{m} \Delta N\right)-T \Delta S_{c}+P\left(A \Delta s_{z}-V_{m} \Delta N\right)$

where $\Delta E$ is the change of the system energy simulation box in reference to maximally separated layers, $E_{m}$ is the molar energy of $\mathrm{CH}_{4}$ molecules, calculated based on the bulk $\mathrm{CH}_{4}$ simulation, $\Delta \mathrm{N}$ is the difference in the number of $\mathrm{CH}_{4}$ molecules with respect to a reference state, $\left(\Delta E-E_{m} \Delta N\right)$ is attributed to the change of energy of gas molecules due to adsorption, $\Delta S_{c}$ is the entropy change for the composite clay plus external gas system, and $V_{m} \Delta N$ is the volume change of the external gas attributed to the change of gas molecules due to adsorption. $V_{m}$ was calculated based on the bulk $\mathrm{CH}_{4}$ simulation.

The calculated system potential energy showed a single, global minimum in the investigated basalspacing range for all models calculated under 1 and 80 bar of $\mathrm{CH}_{4}$, except that of $7.0 \mathrm{H}_{2} \mathrm{O} / \mathrm{Mg} \_\mathrm{Mtm}$ under 1 bar (Fig. S1 A-C). In contrast, the 7.0 $\mathrm{H}_{2} \mathrm{O} / \mathrm{Mg}_{-} \mathrm{Mtm}$ model showed one local (at $\sim 12.1 \AA$ ) and one global (at $\sim 13.5 \AA$ ) minima. An increase of $\mathrm{CH}_{4}$ pressure resulted in a decrease of the system potential energy, especially for Mg_Mtm models. Significant flattening of the system potential energy minimum resulted for $4.5 \mathrm{H}_{2} \mathrm{O} / \mathrm{Mg} \_\mathrm{Mtm}$ (Fig. S1 B) and the removal of local 
system potential energy minima at $12.1 \AA$ for $7.0 \mathrm{H}_{2} \mathrm{O} / \mathrm{Mg} \_$Mtm (Fig. S1 C). For the Cs_Mtm model, the flattening of the energy minimum was not observed (Fig. S1 C).

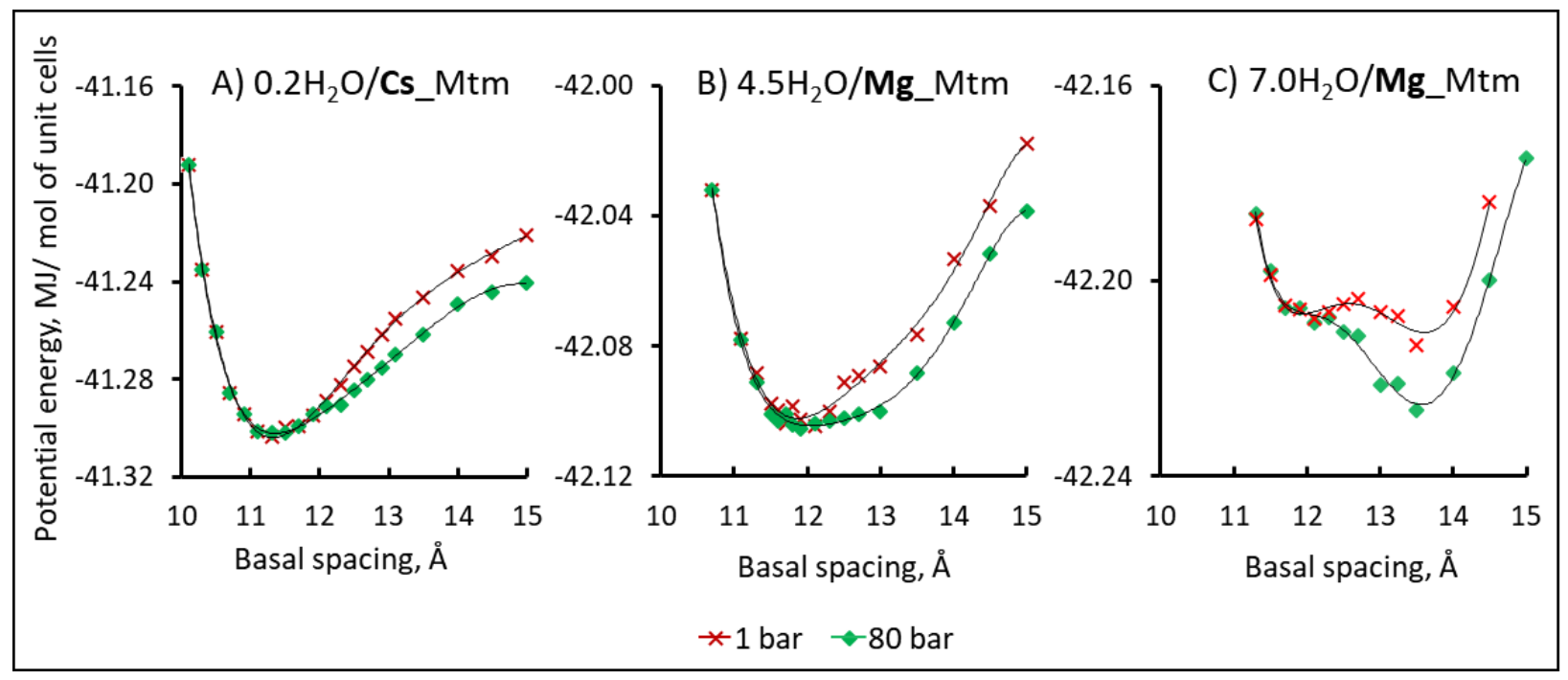

Figure S1. Potential energy with fitted polynomial of $6^{\text {th }}$ order of A) Cs-exchanged montmorillonite models, B) Mg-exchanged montmorillonite models with $4.5 \mathrm{H}_{2} \mathrm{O}$ molecules per $\mathrm{Mg}^{2+}$, and C) $\mathrm{Mg}$ exchanged montmorillonite models with $7.0 \mathrm{H}_{2} \mathrm{O}$ molecules per $\mathrm{Mg}^{2+}$ as a function of basal spacing at 1 and 80 bar of $\mathrm{CH}_{4}$. 
GCMC: Density profiles along the $[00 l]$ direction in the interlayer

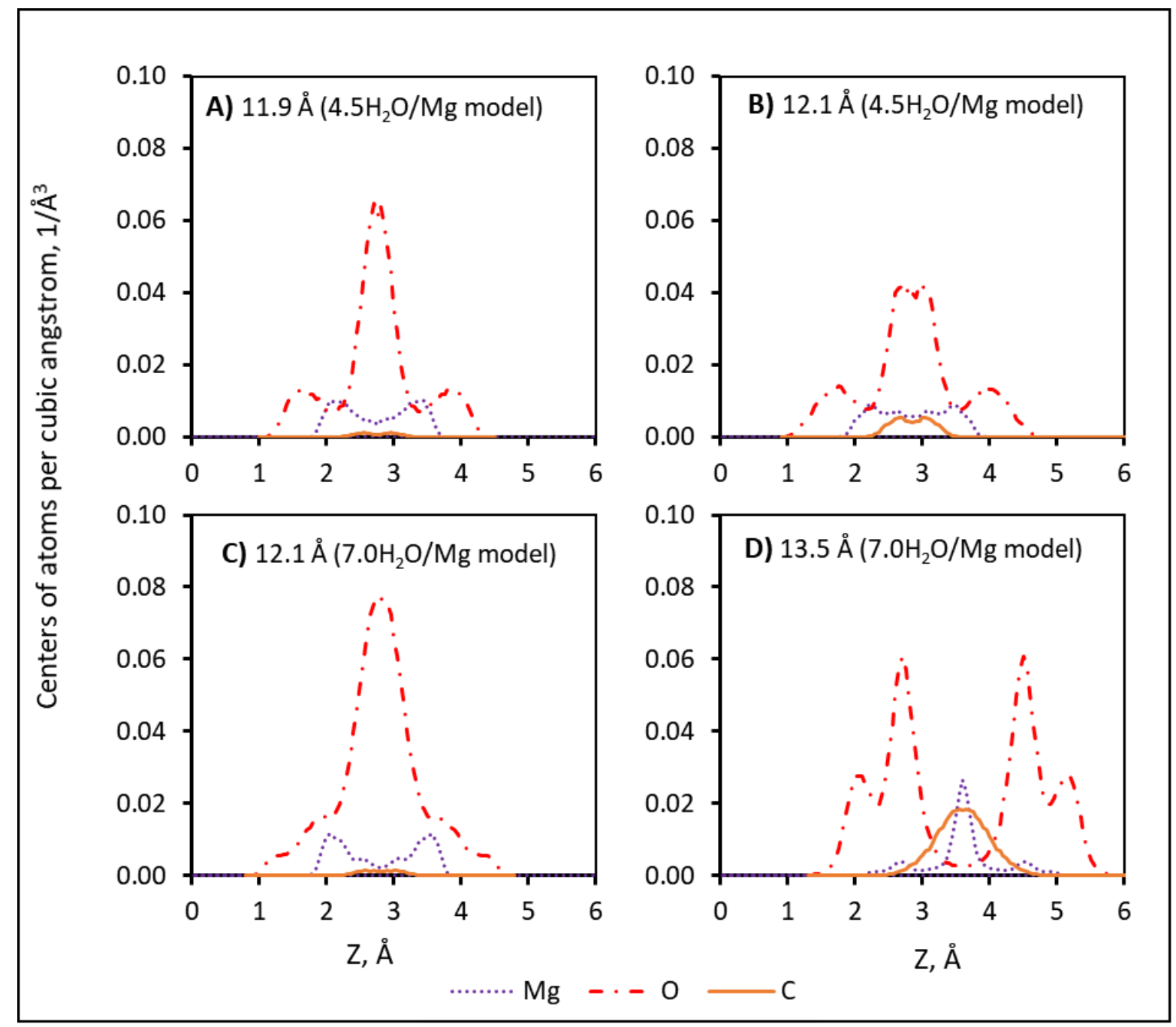

Figure S2. Density profiles of magnesium, oxygen, and carbon atoms centers within montmorillonite interlayers at 80 bar of $\mathrm{CH}_{4}$, based on the GCMC simulation of $\mathrm{Mg}$ montmorillonite containing: $4.5 \mathrm{H}_{2} \mathrm{O} / \mathrm{Mg}^{2+}$ for A) $11.9 \AA$ and B) $12.1 \AA$ basal spacing, and $7.0 \mathrm{H}_{2} \mathrm{O} / \mathrm{Mg}^{2+}$ for C) $12.1 \AA$ and D) $13.5 \AA$ basal spacing. Zero on the $\mathrm{x}$-axis is the average position of oxygen centers in the tetrahedral sheet. 


\section{GCMC: Example snapshots}

A)

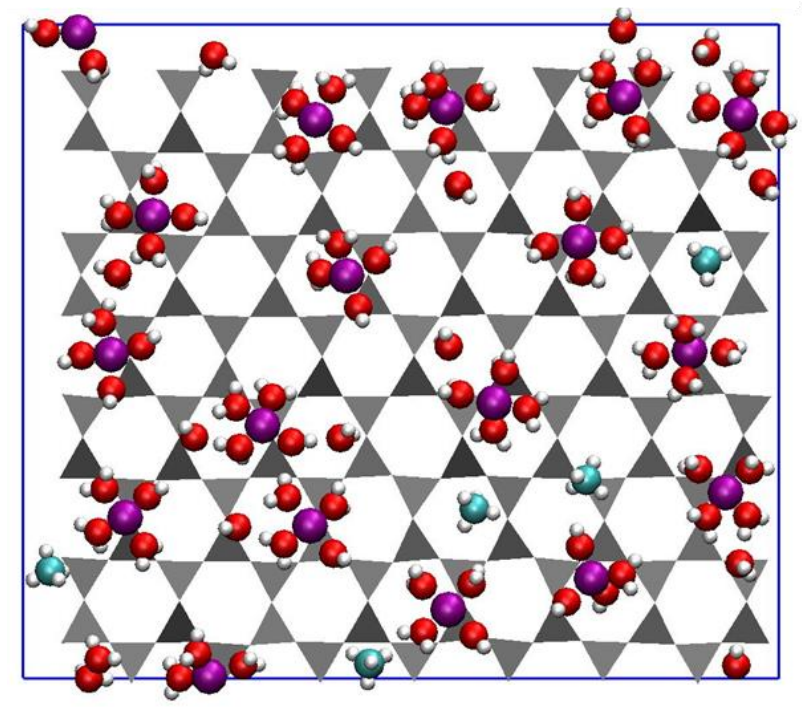

$\mathrm{Mg}^{2+} \mathrm{H}_{2} \mathrm{O}$
B)

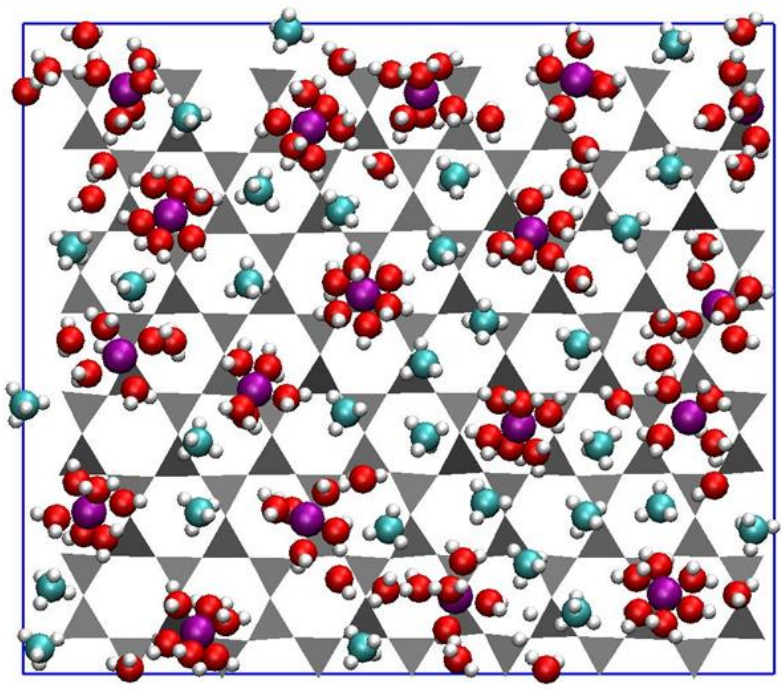

$\mathrm{CH}_{4}$

Figure S3. Snapshots of the GCMC simulation box under 80 bar of $\mathrm{CH}_{4}$ and containing the $\mathrm{Mg}$ montmorillonite model: A) containing $4.5 \mathrm{H}_{2} \mathrm{O} / \mathrm{Mg}^{2+}$ and $12.1 \AA$ basal spacing and B) model containing $7.0 \mathrm{H}_{2} \mathrm{O} / \mathrm{Mg}^{2+}$ and $13.5 \AA$ basal spacing. Methane is never located in the first coordination sphere of hydrated $\mathrm{Mg}^{2+}$ ions. $\mathrm{CH}_{4}$ is adsorbed in the voids between hydrated $\mathrm{Mg}^{2+}$ ions. 
GCMC: Radial distribution functions - $\mathrm{H}_{2} \mathrm{O}$ oxygen and $\mathrm{CH}_{4}$ carbon and $\mathrm{H}_{2} \mathrm{O}$ hydrogen and $\mathrm{CH}_{4}$ carbon
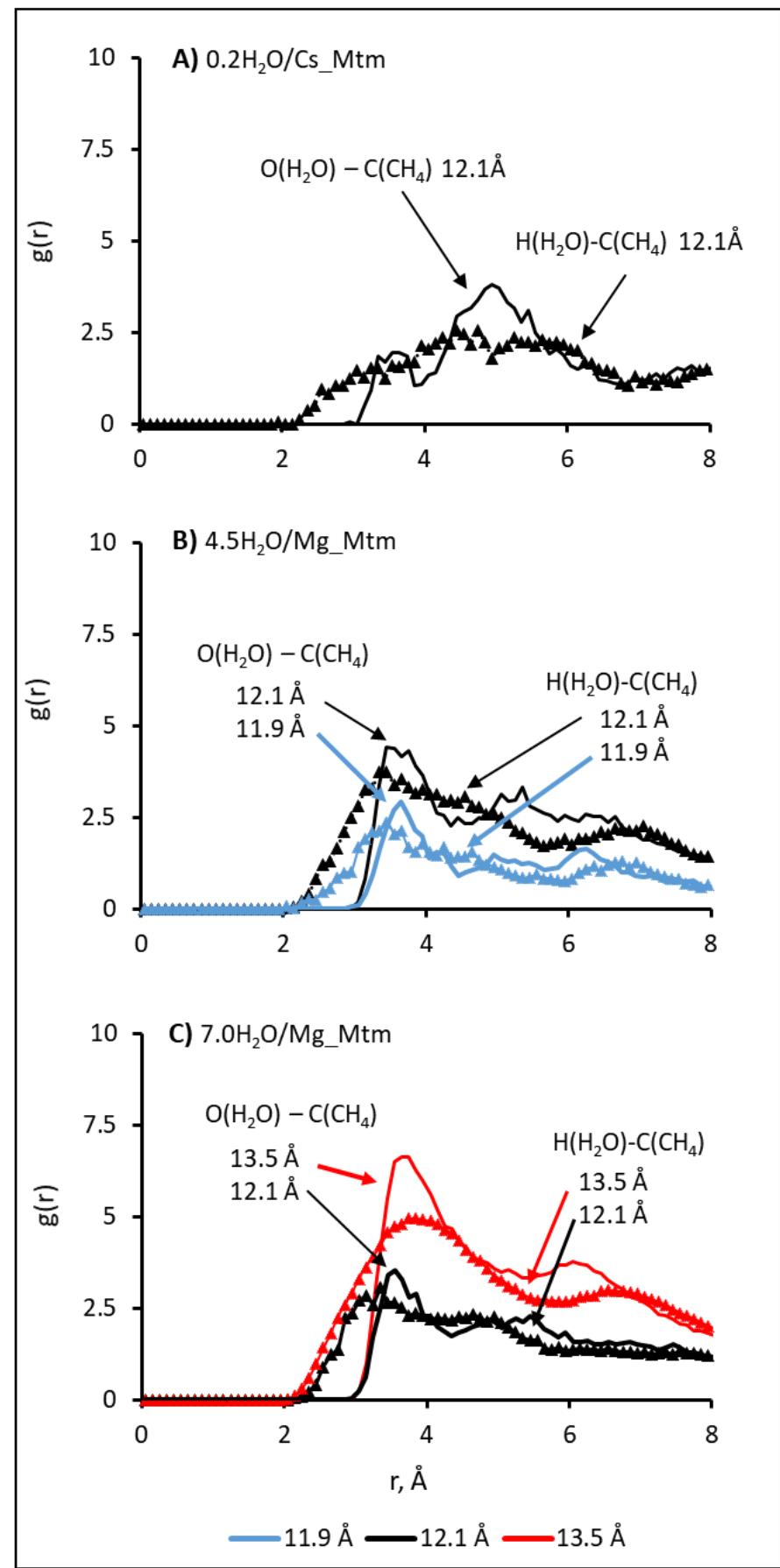

Figure S4. Radial distribution function $[\mathrm{g}(\mathrm{r})]$ between the $\mathrm{H}_{2} \mathrm{O}$ oxygen and $\mathrm{CH}_{4}$ carbon (solid lines) and $\mathrm{H}_{2} \mathrm{O}$ hydrogen and $\mathrm{CH}_{4}$ carbon (triangles). Basal spacings are distinguished by color-coding. 


\section{REFERENCES}

(1) Martin, M. G. MCCCS Towhee: A Tool for Monte Carlo Molecular Simulation. Mol. Simul. 2013, 39 (14-15), 1212-1222.

(2) Deng, L.-Y. Efficient and Portable Multiple Recursive Generators of Large Order. ACM Trans. Model. Comput. Simul. 2005, 15 (1), 1-13.

(3) Lee, J. H.; Guggenheim, S. Single-Crystal X-Ray Refinement of Pyrophyllite-1Tc. Am. Mineral. 1981, 66 (3-4), 350.

(4) Mermut, A. R.; Cano, A. F. Baseline Studies of the Clay Minerals Socienty Source Clays: Chemical Analyses of Major Elements. Clays Clay Miner. 2001, 49 (5), 381-386.

(5) Ortega-Castro, J.; Hernandez-Haro, N.; Dove, M. T.; Hernandez-Laguna, A.; Sainz-Diaz, C. I. Density Functional Theory and Monte Carlo Study of Octahedral Cation Ordering of $\mathrm{Al} / \mathrm{Fe} / \mathrm{Mg}$ Cations in Dioctahedral 2:1 Phyllosilicates. Am. Mineral. 2010, 95 (2-3), 209220.

(6) Cygan, R. T.; Liang, J.-J.; Kalinichev, A. G. Molecular Models of Hydroxide, Oxyhydroxide, and Clay Phases and the Development of a General Force Field. J. Phys. Chem. B 2004, 108 (4), 1255-1266.

(7) Chen, B.; Siepmann, J. I. Transferable Potentials for Phase Equilibria. 3. Explicit-Hydrogen Description of Normal Alkanes. J. Phys. Chem. B 1999, 103 (25), 5370-5379.

(8) Diestler, D. J.; Schoen, M.; Curry, J. E.; Cushman, J. H. Thermodynamics of a Fluid Confined to a Slit Pore with Structured Walls. J. Chem. Phys. 1994, 100 (12), 9140-9146.

(9) Whitley, H. D.; Smith, D. E. Free Energy, Energy, and Entropy of Swelling in Cs-, Na-, and Sr-Montmorillonite Clays. J. Chem. Phys. 2004, 120 (11), 5387-5395.

(10) Shroll, R. M.; Smith, D. E. Molecular Dynamics Simulations in the Grand Canonical Ensemble: Application to Clay Mineral Swelling. J. Chem. Phys. 1999, 111 (19), 90259033.

(11) Smith, D. E.; Wang, Y.; Whitley, H. D. Molecular Simulations of Hydration and Swelling in Clay Minerals. Fluid Phase Equilib. 2004, 222-223 (July), 189-194. 